\title{
Frecuencia de síndrome metabólico en niños con obesidad diagnosticado con criterios de IDEFICS
}

\section{Frequency of metabolic syndrome in children with obesity diagnosed with IDEFICS criteria}

\author{
Mauricio López-Acevedo, ${ }^{*, \ddagger}$ María de la Cruz Ruiz-Jaramillo*,§ \\ * Hospital General León; ${ }^{\ddagger}$ Universidad de Guanajuato; ${ }^{\S}$ Colegio de Pediatras del Estado de Guanajuato \\ capítulo León. México.
}

\begin{abstract}
RESUMEN
Introducción: La iniciativa europea Identification and prevention of Dietary- and lifestyle- induced health EFects In Children and infantS (IDEFICS) estableció criterios para el diagnóstico de síndrome metabólico (SM) en niños menores de 10 años de edad. Objetivo: Describir la frecuencia de SM con los criterios de IDEFICS en niños de tres a 10 años con obesidad (índice de masa corporal $\geq$ percentil 95). Material y métodos: Estudio descriptivo, observacional y retrospectivo realizado entre 2017 y 2018 en el servicio de Pediatría del Hospital General León, Guanajuato, México. Resultados: Se incluyeron 73 pacientes, de los cuales 53 (72.6\%) cumplieron con tres o más criterios de IDEFICS, por lo que se identificaron con SM; de los cuales, 14 pacientes tuvieron nivel de monitorización y 39 nivel de intervención. La dislipidemia y resistencia a la insulina fueron las principales variables alteradas. Conclusiones: El criterio IDEFICS es de utilidad para identificar niños menores de 10 años que tienen uno o más componentes de SM.
\end{abstract}

Palabras clave: Síndrome metabólico, preescolares, escolares, obesidad, dislipidemia, IDEFICS.

\section{INTRODUCCIÓN}

El síndrome metabólico (SM) es una patología cuya epidemiología ha cambiado en los últimos años, se

\begin{abstract}
Introduction: The European initiative: Identification and prevention of Dietary- and lifestyle-induced health EFects In Children and infantS (IDEFICS) established criteria for the diagnosis of metabolic syndrome (MS) in children younger than 10 years of age. Objective: To describe the frequency of MS with the IDEFICS criteria in children aged 3 to 10 years with obesity (body mass index $\geq 95$ th percentile). Material and methods: Descriptive, observational, and retrospective study, carried out between 2017 and 2018 in the Servicio de Pediatría del Hospital General León, Guanajuato, México. Results: A total of 73 patients were included, of which 53 (72.6\%) met three or more IDEFICS criteria, thus being identified with MS. Of the 53 patients, 14 had a monitoring level and 39 had an intervention level. Dyslipidemia and insulin resistance were the most common MS components. Conclusions: The IDEFICS criteria are useful for identifying children younger than 10 years who have one or more SM components.
\end{abstract}

Keywords: Metabolic syndrome, preschoolers, schoolchildren, obesity, dyslipidemia, IDEFICS.

presenta en edades cada vez más tempranas. Se han descrito más de 40 definiciones para este síndrome en niños, ${ }^{1,2}$ siendo las de Cook y Ferranti las más utilizadas. $^{3}$ Sin embargo, en la actualidad el criterio de la

Correspondencia: María de la Cruz Ruiz-Jaramillo, E-mail: maricruiz@gmail.com

Citar como: López-Acevedo M, Ruiz-Jaramillo MC. Frecuencia de síndrome metabólico en niños con obesidad diagnosticado con criterios de IDEFICS. Rev Mex Pediatr. 2021; 88(5): 184-188. https://dx.doi.org/10.35366/103897 
Federación Internacional de Diabetes (IDF, por sus siglas en inglés) es uno de los más aceptados, pero sólo es aplicable a niños de 10 años en adelante. ${ }^{4}$

Desde el año 2014, una iniciativa europea publicó criterios para diagnóstico y manejo de SM en menores de 10 años. Esta iniciativa se denomina Identification and prevention of Dietary-and lifestyle-induced health EFects In Children and infantS (IDEFICS), e incluye ocho países: Suecia, Alemania, Hungría, Italia, Chipre, España, Bélgica y Estonia. Al estudiar una cohorte de 16,228 niños se construyeron valores percentiles para cada componente del SM. De acuerdo con los percentiles, se consideraron dos grados de SM: 1) percentil 90 para circunferencia de cintura, presión arterial, triglicéridos, glucosa y Homeostasis Model Assessment Index (índice HOMA), así como el percentil 10 para lipoproteínas de alta densidad (HDL) como el punto de corte para diagnosticar SM en grado de monitorización. 2) Para el grado de intervención se consideró: percentil $<5$ para HDL, y percentil > 95 para el resto de los parámentros. Señalan que la monitorización implica promoción de estilo de vida saludable así como evaluaciones periódicas. Por su parte, el grado de intervención consiste en otorgar medidas terapéuticas por un equipo multidisciplinario. Cuando compararon la frecuencia del SM, se observó que con la definición de la IDF la prevalencia fue menor que con la IDEFICS (5.5\% versus $0.4 \%){ }^{5}$

Hasta la fecha no se han identificado estudios publicados con la clasificación IDEFICS fuera de Europa; para su aplicación en cualquier paciente existe una calculadora de libre acceso en Internet: ${ }^{6}$ https:// mets-score.bips.eu/

En el estado de Guanajuato, México, la prevalencia de obesidad entre cinco y 11 años es de $45.1 \%,{ }^{7}$ lo que supera la media nacional de $35.5 \%$. El objetivo de este estudio es estimar la frecuencia de SM en niños preescolares y escolares con obesidad, de acuerdo con los criterios diagnósticos de la IDEFICS.

\section{MATERIAL Y MÉTODOS}

Entre enero de 2017 y diciembre de 2018 se realizó un estudio transversal y retrospectivo con niños atendidos en la consulta externa del servicio de Pediatría del Hospital General León, en Guanajuato, México. Los pacientes acudieron para atención de diferentes patologías y se les aplicó el algoritmo de atención clínica para identificar casos de sobrepeso y obesidad. ${ }^{8}$

Se incluyeron pacientes ambos sexos, con edad de tres a 10 años con 11 meses, con índice de masa cor- poral $(\mathrm{IMC}) \geq 95$. Se excluyeron quienes presentaban malformaciones congénitas o pacientes con tratamiento esteroideo.

Las variables de estudio fueron: edad, sexo, peso, talla, IMC, circunferencia de cintura, presión arterial así como niveles séricos de triglicéridos, colesterol HDL, glucosa e insulina en ayuno. Se calculó el índice HOMA con la fórmula: [glucosa $(\mathrm{mg} / \mathrm{dL}) \times$ insulina (microU/ $\mathrm{mL}$ )]/405. Se usó metodología de ISAK (International Society for the Advancement of Kinanthropometry) para la somatometría con un solo evaluador. La medición de presión arterial se realizó con baumanómetro aneroide utilizando brazaletes de acuerdo con la longitud del brazo de cada paciente. Las mediciones de glucosa, triglicéridos y colesterol HDL se realizaron con la técnica de espectrofotometría automatizada, y la insulina sérica mediante electroquimioluminiscencia.

El diagnóstico de SM se realizó con base en tres o más criterios del IDEFICS, clasificándose en categorías de monitorización o de intervención, utilizando la calculadora automática para ubicar los percentiles de cada variable por paciente. ${ }^{6}$

Se calculó un tamaño de la muestra de 73 pacientes; se consideró el estudio de Ahrens y colaboradores ${ }^{5}$ para una frecuencia de SM de 5.5\%, y una precisión de 5\%, nivel de confianza de $95 \%$ y potencia de $80 \%$.

Análisis estadístico. Los datos se presentan con promedio y desviación estándar para las variables numéricas con distribución normal, y como mediana y rango intercuartil para las variables sin distribución normal. La prueba de Kolmogorov-Smirnov se usó para determinar el tipo de distribución de las variables numéricas. Las variables categóricas se presentan con número absoluto y porcentajes.

Se utilizó el paquete estadístico NCSS 2004.

\section{RESULTADOS}

En la Tabla 1 se muestran las características generales de los 73 de niños con obesidad estudiados. Como se observa, la proporción de hombres y mujeres fue casi igual; por edad, hubo pacientes desde tres y hasta 10 años, siendo la mediana de ocho. En cuanto al peso para le edad, la mayoría se encontraba en percentil > 95, mientras que para el IMC $38.4 \%(\mathrm{n}=31)$ estaba en percentil $\geq 99$.

De acuerdo con la clasificación de IDEFICS, la frecuencia de SM fue 53 casos (73\%), de los cuales 39 pacientes tenían nivel de intervención, y 14 tenían nivel de monitorización. 
Tabla 1: Datos generales de 73 pacientes con obesidad.

$\begin{array}{lc}\text { Variable } & n(\%) \\ \text { Sexo } & \\ \text { Femenino } & 37(50.7) \\ \text { Masculino } & 36(49.3) \\ \text { Edad (años) } & \\ 3 & 5(6.8) \\ 5 & 7(9.6) \\ 6 & 4(5.5) \\ 7 & 9(12.3) \\ 8 & 13(17.8) \\ 9 & 15(20.5) \\ 10 & 20(27.4) \\ \text { Percentil } & \\ \text { Peso } & \\ >95 & 57(78.1) \\ 95 & 6(8.2) \\ 90 & 8(10.9) \\ 75 & 1(1.4) \\ 50 & 1(1.4) \\ \text { Índice de masa corporal } & \\ >99 & 14(19.2) \\ 99 & 17(23.3) \\ 98 & 18(24.7) \\ 97 & 10(13.7) \\ 96 & 4(5.5) \\ 95 & 10(13.7) \\ & \end{array}$

En la Tabla 2 se comparan los tres grupos: dos con SM, y el grupo sin SM. Como era de esperarse, en general las características clínicas y de laboratorio del grupo sin SM fueron mejor que en los otros dos grupos. Sin embargo, no hubo diferencia en los valores de presión arterial y en los de glucemia.

Por último, en la Tabla 3 se compara la frecuencia de los componentes de SM. En los tres grupos se observaron alteraciones, pero predominan las alteraciones en triglicéridos, HDL e índice HOMA. En el grupo SM de nivel de intervención se identificaron casos con los seis componentes.

\section{DISCUSIÓN}

Los resultados de este estudio muestran una frecuencia de SM más alta que el estudio original de IDEFICS $^{9}$ (73 versus 5.5\%), lo cual se relaciona a que en la iniciativa europea se incluyeron niños con y sin obesidad, mientras que en este estudio son exclusivamente niños con obesidad. Pero además, se debe tener en cuenta que el tamaño de muestra es muy limitado, y que existe una amplia variabilidad en la edad de los participantes. En publicaciones más recientes en pacientes prepúberes europeos con obesidad, la frecuencia de SM fue de $26 \%$ con el criterio IDEFICS. ${ }^{10}$

Tabla 2: Datos clínicos y de laboratorio de acuerdo con el nivel de síndrome metabólico comparado con niños sin síndrome metabólico.

\begin{tabular}{|c|c|c|c|}
\hline \multirow[b]{2}{*}{ Variable } & \multicolumn{3}{|c|}{ Síndrome metabólico } \\
\hline & Sin sindrome $(\mathbb{N}=20)$ & Nivel monitorización ( $\mathrm{N}=14$ ) & Nivel intervención ( $\mathrm{N}=39$ ) \\
\hline Edad (años)* & $7.5(5-9)$ & $9(7-10)$ & $9(7-10)$ \\
\hline Sexo (femenino/masculino) $\ddagger$ & $8 / 12$ & $8 / 6$ & $21 / 18$ \\
\hline Peso $(\mathrm{kg})^{\S}$ & $35.5 \pm 9.5$ & $42.5 \pm 10.3$ & $47 \pm 13.0$ \\
\hline Talla $(\mathrm{cm})^{\S}$ & $125 \pm 14.0$ & $134 \pm 16.0$ & $137 \pm 12.0$ \\
\hline IMC $\left(\mathrm{kg} / \mathrm{m}^{2}\right)^{\S}$ & $22.2 \pm 2.5$ & $23.3 \pm 1.9$ & $24.7 \pm 3.8$ \\
\hline Cintura $(\mathrm{cm})^{\S}$ & $71.4 \pm 8.0$ & $77.3 \pm 6.8$ & $82.2 \pm 10.1$ \\
\hline Presión sistólica $(\mathrm{mmHg})^{\S}$ & $92.5 \pm 12.5$ & $97 \pm 10.0$ & $99 \pm 14.6$ \\
\hline Presión diastólica $(\mathrm{mmHg})^{*}$ & $53 \pm 9.0$ & $55 \pm 8.5$ & $60 \pm 11.0$ \\
\hline Triglicéridos $(\mathrm{mg} / \mathrm{dL})^{*}$ & $79.5(68-103.5)$ & $100.5(82-106.5)$ & $159(137-225)$ \\
\hline $\mathrm{HDL}(\mathrm{mg} / \mathrm{dL})^{\S}$ & $43.8 \pm 11.6$ & $40.1 \pm 10.4$ & $36.7 \pm 9$ \\
\hline Glucosa (mg/dL)* & $85(80-88.0)$ & $85(79.5-89.0)$ & $87(82-95)$ \\
\hline Insulina $(\mu \mathrm{U} / \mathrm{mL})^{*}$ & $7.3(5.8-10.0)$ & $14.1(9.6-17.4)$ & $15.7(12.9-28.2)$ \\
\hline Índice HOMA* & $1.5(1.2-2.1)$ & $2.8(2.1-3.6)$ & $3.3(2.8-6.7)$ \\
\hline
\end{tabular}

IMC = índice de masa corporal; HDL = lipoproteína de alta densidad; HOMA = Homeostasis Model Assessment Index.

${ }^{\star}$ Mediana (rango intercuartil), ${ }^{\ddagger} \mathrm{n}(\%), \S$ promedio, desviación estándar. 
Tabla 3: Porcentaje de pacientes con valores anormales de cada criterio de acuerdo al nivel de síndrome metabólico.

\begin{tabular}{|c|c|c|c|}
\hline \multirow[b]{2}{*}{ Criterio } & \multicolumn{3}{|c|}{ Síndrome metabólico, \% } \\
\hline & $\begin{array}{l}\text { Sin síndrome } \\
\qquad(N=20)\end{array}$ & $\begin{array}{c}\text { Nivel de } \\
\text { monitoriza- } \\
\text { ción }(\mathbb{N}=14)\end{array}$ & $\begin{array}{c}\text { Nivel de } \\
\text { intervención } \\
(\mathrm{N}=39)\end{array}$ \\
\hline Adiposidad central & 100 & 100 & 100 \\
\hline $\begin{array}{l}\text { Hipertensión } \\
\text { arterial sistólica }\end{array}$ & 5.0 & 0.0 & 18.0 \\
\hline $\begin{array}{l}\text { Hipertensión } \\
\text { arterial diastólica }\end{array}$ & 0.0 & 0.0 & 13.0 \\
\hline Hipertrigliceridemia & 30.0 & 71.5 & 92.0 \\
\hline Colesterol HDL bajo & 20.0 & 50.0 & 54.0 \\
\hline Índice HOMA alto & 25.0 & 85.5 & 97.5 \\
\hline
\end{tabular}

HDL = lipoproteína de alta densidad.

En el presente estudio, la prevalencia de SM también fue mayor al $62 \%$ reportado por Evia Visca$\mathrm{rra}^{11}$ en el 2013, en el que se incluyeron niños mexicanos de ocho a 15 años con obesidad, utilizando los criterios Adult Treatment Panel III (ATP III).

En comparación con los criterios de IDF mediante el uso de IDEFICS se puede tamizar a niños menores de 10 con obesidad para determinar si tienen uno o más de los componentes de SM. Con los datos de este estudio podemos afirmar que el criterio IDEFICS es útil en población mexicana, lo cual debería ayudar a la referencia oportuna para otorgar medidas encaminadas a mejorar su perfil metabólico. Desde la primera publicación de la iniciativa IDEFICS en 2014, estos criterios se han difundido para identificar e intervenir tempranamente en los niños con SM. Las intervenciones más importantes que sugieren son cambios en aspectos nutricionales así como el control de tiempo frente a la pantalla, y calidad de sueño. ${ }^{9,12-15}$

Un punto a destacar es que el índice HOMA requiere la medición de insulina sérica, lo cual no está disponible en muchas unidades médicas de primer nivel de atención, por lo que se podría usar el índice triglicéridos/HDL, que ha mostrado buena correlación con resistencia a la insulina. ${ }^{16}$

Nuestro estudio presenta debilidades por ser un diseño retrospectivo, pero tiene la fortaleza de que los datos clínicos y de laboratorio fueron obtenidos de manera prospectiva y sistemática de acuerdo al algoritmo de atención clínica para niños con sobre- peso y obesidad del Plan Estratégico Sectorial para la Difusión e Implementación de Guías de Práctica Clínica ${ }^{8,17,18}$

\section{CONCLUSIONES}

El criterio IDEFICS en niños menores de 10 años con obesidad es una herramienta útil para identificar casos de SM.

\section{AGRADECIMIENTOS}

Agradecimiento al personal del Servicio de Pediatría y al Departamento de Laboratorio Clínico del Hospital General León para la realización de este estudio.

\section{REFERENCIAS}

1. Eyzaguirre F, Silva R, Román R, Palacio A, Cosentino M, Vega V et al. Prevalencia de síndrome metabólico en niños y adolescentes que consultan por obesidad. Rev Med Chile. 2011; 139: 732-738.

2. Wittcopp C, Conroy R. Metabolic syndrome in children and adolescents. Pediatr Rev. 2016; 37(5): 193-202. doi: 10.1542/ pir.2014-0095.

3. Cornejo-Barrera J, Llanas-Rodríguez JD. Epidemiología del síndrome metabólico en pediatría. Evid Med Invest Salud. 2013; 6(3): 89-94.

4. Burguete-García AI, Valdés-Villalpando YN, Cruz M. Definiciones para el diagnóstico de síndrome metabólico en población infantil. Gac Med Mex. 2014; 150(s1): 79-87.

5. Ahrens W, Moreno LA, Marild S et al. Metabolic syndrome in young children: definitions and results of the IDEFICS study. Int $J$ Obes (Lond). 2014; 38 Suppl 2: S4-S14. doi: 10.1038/ijo.2014.130.

6. Bundesministerium für Gesundheit. Deutschlands Initiative für gesunde Ernahrung und mehr Bewegung. IDEFICS Metabolic Syndrome Score Tool. Identification and prevention of Dietary and lifestyle-induced health effects in Children and Infants. Duitsland. Available in: https://mets-score.bips.eu

7. Secretaría de Salud. Encuesta Nacional de Salud y Nutrición 2018. Informe de resultados por entidad federativa. México: Instituto Nacional de Estadística y Geografía, Instituto Nacional de Salud Pública; 2019. Disponible en: https://ensanut.insp.mx/encuestas/ ensanut2018/doctos/informes/Resultado_Entidad_Guanajuato.pdf

8. Secretaría de Salud. Gobierno de México. Algoritmos de atención clínica. Sobrepeso y obesidad en niños y adolescentes. México: 2018. Disponible en: http://www.calidad.salud.gob.mx/site/calidad/ docs/algoritmo_sobrepeso_adolescentes.pdf

9. Nappo A, González-Gil EM, Ahrens W et al. Analysis of the association of leptin and adiponectin concentrations with metabolic syndrome in children: results from the IDEFICS study. Nutr Metab Cardiovasc Dis. 2017; 27(6): 543-551. doi: 10.1016/j. numecd.2017.04.003.

10. Bornhorst C, Russo P, Veidebaum T et al. Metabolic status in children and its transitions during childhood and adolescence-the IDEFICS/I. Family study. Int J Epidemiol. 2019; 48(5): 1673-1683. doi: 10.1093/ije/dyz097.

11. Evia-Viscarra ML, Rodea-Montero ER, Apolinar-Jiménez E, Quintana-Vargas S. Metabolic syndrome and its components 
among obese (BMI $>=95$ th) Mexican adolescents. Endocr Connect. 2013; 2(4): 208-215. doi: 10.1530/EC-13-0057.

12. Peplies J, Jiménez-Pavón D, Savva SC et al. Percentiles of fasting serum insulin, glucose, HbA1c and HOMA-IR in pre-pubertal normal weight European children from the IDEFICS cohort. Int J Obes (Lond). 2014; 38 Suppl 2: S39-S47. doi: 10.1038/ ijo.2014.134.

13. Nagrani R, Foraita R, Gianfagna F et al. Common genetic variation in obesity, lipid transfer genes and risk of metabolic syndrome: results from IDEFICS/I. Family study and metaanalysis. Sci Rep. 2020; 10(1): 7189. doi: 10.1038/s41598020-64031-2.

14. Iglesia I, Intemann T, De Miguel-Etayo P et al. Dairy consumption at snack meal occasions and the overall quality of diet during childhood. Prospective and Cross-sectional analyses from the IDEFICS/I. Family cohort. Nutrients. 2020; 12(3): 642. doi: 10.3390/ nu12030642.

15. Santaliestra-Pasías AM, González-Gil EM, Pala V et al. Predictive associations between lifestyle behaviours and dairy consumption: The IDEFICS study. Nutr Metab Cardiovasc Dis. 2020; 30(3): 514-522. doi: 10.1016/j.numecd.2019.10.006.

16. Quijada Z, Paoli M, Zerpa Y, Camacho N, Cichetti R, Villarroel $\mathrm{V}$, et al. The triglyceride/HDL-cholesterol ratio as a marker of cardiovascular risk in obese children; association with traditional and emergent risk factors. Pediatr Diabetes. 2008 Oct;9(5):464-71. doi: 10.1111/j.1399-5448.2008.00406.x.

17. Secretaría de Salud. Encuesta Nacional de Salud y Nutrición 2018. México: Instituto Nacional de Estadística y Geografía, Instituto Nacional de Salud Pública: 2019. Disponible en: https://ensanut. insp.mx/encuestas/ensanut2018/doctos/informes/ensanut_2018_ presentacion resultados.pdf

18. Secretaría de Salud Subsecretaría de Prevención y Promoción de la Salud. Declaratoria de Emergencia Epidemiológica EE-3-2016. Ciudad de México: Centro Nacional de Programas Preventivos y Control de Enfermedades; 2016. Disponible en: https://www.gob. $\mathrm{mx} / \mathrm{cms} /$ uploads/attachment/file/265571/EE_3.pdf

Aspectos éticos: Para la realización de este estudio se obtuvo autorización del comité de ética en investigación del hospital, con el número de registro SSGTO0171. Dado que el estudio fue a partir de los datos del expediente clínico, no se requirió consentimiento $o$ asentimiento informado.

Conflicto de intereses: Los autores declaran que no tienen. 